\title{
ON THE CHARACTERIZABILITY OF SOME FAMILIES OF FINITE GROUP OF LIE TYPE BY ORDERS AND VANISHING ELEMENT ORDERS
}

\author{
Majedeh Pasdar and Ali Iranmanesh
}

(C) 2019 by University of Niš, Serbia | Creative Commons Licence: CC BY-NC-ND

\begin{abstract}
In this paper, we show that the following simple groups are uniquely determined by their orders and vanishing element orders: $A_{p-1}(2)$, where $p \neq 3,{ }^{2} D_{p+1}(2)$, where $p \geq 5, p \neq 2^{m}-1, A_{p}(2), C_{p}(2), D_{p}(2), D_{p+1}(2)$ which for all of them $p$ is an odd prime and $2^{p}-1$ is a Mersenne prime. Also, ${ }^{2} D_{n}(2)$ where $2^{n-1}+1$ is a Fermat prime and $n>3,{ }^{2} D_{n}(2)$ and $C_{n}(2)$ where $2^{n}+1$ is a Fermat prime. Then we give an almost general result to recognize the non-solvability of finite group $H$ by an analogy between orders and vanishing element orders of $H$ and a finite simple group of Lie type. Keywords: simple groups; Mersenne prime; Fermat prime; Lie group.
\end{abstract}

2010 Mathematics Subjedct Classification: 20C15; 20 D05.

Keywords: Finite simple groups, vanishing element orders, prime graph.

\section{Introduction}

Throughout this paper $G$ and $H$ are two finite groups. Let $X$ be a finite set of positive integers. The prime graph $\Pi(X)$ is a graph whose vertices are the prime divisors of elements of $X$, and two distinct vertices $p$ and $q$ are adjacent if there exists an element of $X$ divisible by $p q$. For a finite group $G$, we denote by $\omega(G)$, the set of element orders of $G$. The prime graph $\Pi(\omega(G))$ is denoted by $G K(G)$ and is called the Gruenberg-Kegel graph of $G$. Here, $s(G)$ denotes the number of connected components of $G K(G)$. For the group $G$, we denote by $\rho(G)$ some independence sets in $G K(G)$ with maximal number of vertices and put $t(G)=|\rho(G)|$, independence number of $G K(G) . g \in G$ is called a vanishing element of $G$ if $\chi(g)=0$ for some $\chi \in \operatorname{Irr}(G)$. Let us denote by $\operatorname{Van}(G)$ and $\operatorname{vo}(G)$ the set of all vanishing elements

Received January 09, 2019; accepted March 13, 2019

2010 Mathematics Subject Classification. Primary 20C15; Secondary 20D05 
and the set of vanishing element orders of $G$, respectively. Also the prime graph $\Pi(\operatorname{vo}(G))$ is denoted by $\Gamma(G)$ and is called the vanishing prime graph of $G$.

If $n$ is a natural number and $\pi$ is a set of primes, then we denote the set of all prime divisors of $n$ by $\pi(n)$, and the maximal divisor $t$ of $n$ such that $\pi(t) \subseteq \pi$ by $n_{\pi}$. If $\pi(G)$ is the set of prime divisors of $|G|$, then $\pi_{i}(G)=\pi\left(m_{i}\right)$ for some positive integers $m_{i}, 1 \leq i \leq t$, such that $|G|=m_{1} m_{2} \cdots m_{t}$ and $t=s(G)$. Also for any group with even order, $2 \in \pi_{1}(G)$. We set $O C(G)=\left\{m_{1}, \cdots, m_{t}\right\}$ and call the set of order components of $G$. A finite simple group $G$ is said characterizable by its order components, if $G \cong H$ for each finite group $H$ such that $O C(G)=O C(H)$. Some authors have proved that some non-abelian simple groups are recognizable by their order components. We refer the reader to [23] to find a list of papers with the OC-characterizability criterion for some finite simple groups.

It was shown in [38] that if $G$ is a finite group such that $\operatorname{vo}(G)=\operatorname{vo}\left(A_{5}\right)$ then $G \cong A_{5}$. According to this result, M. Foroudi, A. Iranmanesh and F. Mavadatpour in [12] stated the conjecture as follows:

Conjecture 1.1. Let $G$ and $H$ be two groups with the same order. If $G$ is a non-abelian group and $\operatorname{vo}(G)=\operatorname{vo}(H)$, then $G \cong H$.

First, this conjecture was proved for $L_{2}(q)$, where $q \in\{5,7,8,9,17\}, L_{3}(4), A_{7}$, $S z(8)$ and $S z(32)$ in [12]. Then they proved this conjecture in [13] for finite simple $K_{n}$-groups with $n \in\{3,4\}$, sporadics, alternatatings and $L_{2}(p)$ where $p$ is an odd prime. In [24] it has been verified that the groups $S z(q)$ satisfy this conjecture, where $q=2^{2 n+1}$ and either $q-1, q-\sqrt{2 q}+1$ or $q+\sqrt{2 q}+1$ is a prime, and $F_{4}(q)$, where $q=2^{n}$ and either $q^{4}+1$ or $q^{4}-q^{2}+1$ is a prime. In this paper, we show that the above conjecture is valid for some families of simple groups of Lie type. Then we prove another result about non-solvability of some finite group using vanishing element orders. In fact, we prove the following theorems:

Theorem 1.1. Let $G$ and $H$ be two groups with the same order and $G$ be a simple group of Lie type $A_{p-1}(2)$ where $p \neq 3,{ }^{2} D_{p+1}(2)$, where $p \geq 5, p \neq 2^{m}-1, A_{p}(2)$, $C_{p}(2), D_{p}(2), D_{p+1}(2)$, which for all of them $p$ is an odd prime and $2^{p}-1$ is a Mersenne prime, ${ }^{2} D_{n}(2)$ where $2^{n-1}+1$ is a Fermat prime, ${ }^{2} D_{n}(2)$ and $C_{n}(2)$ where for the last two groups $2^{n}+1$ is a Fermat prime. If $\operatorname{vo}(G)=\operatorname{vo}(H)$, then $G \cong H$.

Theorem 1.2. Let $G$ and $H$ be two groups with the same order. Suppose $G$ is a simple group of Lie type with $s(G) \geq 2$ except $A_{2}(q)$, where $(q-1)_{3} \neq 3, q$ is a Mersenne prime, ${ }^{2} A_{2}(q)$, where $(q+1)_{3} \neq 3, q$ is a Fermat prime, $C_{2}(q)$ where $q>2$. If $\operatorname{vo}(G)=\operatorname{vo}(H)$, then $H$ is non-solvable.

\section{Preliminaries}

In this section, we state some results which will be of use to the proof of the main theorems. 
Definition 2.1. A group $G$ is said to be a 2-Frobenius group if there exist two normal subgroups $F$ and $L$ of $G$ with the following properties: $L$ is a Frobenius group with kernel $F$, and $G / F$ is a Frobenius group with kernel $L / F$.

Recall that a Frobenius group with kernel $N$ and complement $H$ is a semidirect product $G=H \ltimes N$ such that $N$ is a normal subgroup in $G$, and $C_{N}(h)=1$ for every non-identity element $h$ of $H$. As is well-known, $N$ is the Fitting subgroup of G.

Definition 2.2. $G$ is a nearly 2-Frobenius group if there exists two normal subgroups $F$ and $L$ of $G$ with the following properties: $F=F_{1} \times F_{2}$ is nilpotent, where $F_{1}$ and $F_{2}$ are normal subgroups of $G$, furthermore $G / F$ is a Frobenius group with kernel $L / F, G / F_{1}$ is a Frobenius group with kernel $L / F_{1}$, and $G / F_{2}$ is a 2 -Frobenius group.

Lemma 2.1. [11]

(a) Let $G$ be a solvable Frobenius group with kernel $F$ and complement $H$. The graph $G K(G)$ has two connected components, whose vertex sets are $\pi_{1}=\pi(F)$ and $\pi_{2}=\pi(H)$, and which are both complete graphs.

(b) Let $G$ be a finite solvable group. Then $\Gamma(G)$ has at most two connected components. Moreover, if $\Gamma(G)$ is disconnected, then $G$ is either a Frobenius group or a nearly 2-Frobenius group.

(c) Let $G$ be a nearly 2-Frobenius group. If $\Gamma(G)$ is disconnected, then each connected component is a complete graph.

(d) Let $G$ be a solvable Frobenius group with kernel $F$ and complement $H$. If $F \cap \operatorname{Van}(G) \neq \varnothing$, then $\Gamma(G)=G K(G)$, and the otherwise $\Gamma(G)$ coincides with the connected component of $G K(G)$ with vertex set $\pi(H)$.

Lemma 2.2. [10] If $G$ is a finite non-abelian simple group, then $G K(G)=\Gamma(G)$, unless $G \cong A_{7}$.

Theorem 2.1. [13] Let $G$ be a finite group and let $M$ be a simple $K_{3}$-group or a $K_{4}$-group. If $|G|=|M|$ and $\operatorname{vo}(G)=\operatorname{vo}(M)$, then $G \cong M$.

Recall that a finite simple group $G$ is called a $K_{n}$-group if its order has exactly $n$ distinct prime divisors, where $n$ is a natural number.

Theorem 2.2. [36] Let $G$ be a finite simple group. Then all the connected components of $G K(G)$ are cliques if and only if $G$ is one of the following: $A_{5}, A_{6}, A_{7}$, $A_{9}, A_{12}, A_{13}, M_{11}, M_{22}, J_{1}, J_{2}, J_{3}$, HS, $A_{1}(q)$, with $q>2$, Sz $(q)$ with $q=2^{2 m+1}$, $C_{2}(q), G_{2}\left(3^{k}\right), A_{2}(q)$ where $q$ is a Mersenne prime, ${ }^{2} A_{2}(q)$ where $q$ is a Fermat prime, $A_{2}(4),{ }^{2} A_{2}(9),{ }^{2} A_{3}(3),{ }^{2} A_{5}(2), C_{3}(2), D_{4}(2),{ }^{3} D_{4}(2)$. 


\section{Main results}

To prove Theorem 1.2, we adopt Table I by [14] of components of prime graphs of simple groups of Lie type over a field of even characteristics which in this table $p$ is an odd prime. In Table $1, m_{2}$ coincides with the factor for primes in the second connected component. Table 2 shows $O C$-characterizable groups of Lie type with their prime graph having two connected components. We also use Tables 3 and 4 for the proof of Theorem 1.3. These tables were adopted from [37] and they show the independence number of prime graphs of finite simple groups of Lie type and. In Tables 3 and $4, n$ and $k$ are natural numbers. $[x]$ denotes the integral part of $x$. We assume that $G$ is a finite non-abelian simple group of Lie type over a field of characteristic $p$ and order $q$. We define the primitive prime divisor of $q^{m}-1$ by $r_{m}$. If $p$ is odd then we say that 2 is a primitive prime divisor of $q-1$ if $q \equiv 1(\bmod 4)$ and that 2 is a primitive prime divisor of $q^{2}-1$ if $q \equiv-1(\bmod 4)$.

The following lemma is a conclusion from some noteworthy properties of a simple group $G$ with $s(G)=2$ and the conditions of Conjecture 1.1.

Lemma 3.1. Let $G$ and $H$ be two groups with the same order. Suppose that $G$ is a non-abelian simple group with $s(G)=2$ and $G K(H)$ is disconnected. If $\operatorname{vo}(G)=\operatorname{vo}(H)$, then $O C(G)=O C(H)$.

Proof. The assumption vo $(G)=\operatorname{vo}(H)$ and Lemma 2.2 imply $G K(G)=\Gamma(G)=$ $\Gamma(H)$. So the set of vertices of the vanishing prime graph of $H$ is equal to $\pi(H)$. Since $\Gamma(H) \leq G K(H)$, the prime graph of $H$ has two connected components. Let $O C(G)=\left\{m_{1}, m_{2}\right\}$ and $O C(H)=\left\{n_{1}, n_{2}\right\}$. It is sufficient to prove $m_{1}=n_{1}$. Assume $m_{1} \neq n_{1}$. Therefore, $\pi_{1}(G) \neq \pi_{1}(H)$. Without loss of generality, we suppose there is a prime $p$ in $\pi_{1}(G)$ such that $p \notin \pi_{1}(H)$. So $p \in \pi_{2}(H)$. The connectedness of components implies $\pi_{1}(G) \subseteq \pi_{2}(H)$, that is, $2 \in \pi_{2}(H)$, a contradiction. If $p$ is an isolated vertex, then $p=2$ because the order of $G$ is even. Therefore $2 \in \pi_{2}(H)$ which is impossible.

Before bringing forward the proof of Theorem 1.2, we recall that an irreducible character $\chi$ of group $G$ is called $p$-defect zero if $p \nmid|G| / \chi(1)$ where $p$ is a prime.

\subsection{Proof of Theorem 1.2}

First we show that $G K(H)$ is disconnected. According to Table $1, s(G)=2$ and the second order component of $G$ are prime. From vo $(G)=\operatorname{vo}(H)$ and Lemma 2.2 , we deduce $G K(G)=\Gamma(G)=\Gamma(H)$. The last equalities imply that $\Gamma(H)$ has a connected component with a single vertex $p$. On the other hand, $H$ has a vanishing $p$-element. Since characters of degree not divisible by some prime number $p$ never vanish on $p$-elements, it is then clear that $H$ has a $p$-defect zero character, namely $\chi$. We claim that $G K(H)$ is disconnected. We assume the assertion is false. Then there exists a non-vanishing element $x$ of order $p q$ in $H$ where $q \in \pi_{1}(G)$. Since any $p$-defect zero characters vanish on elements of order divisible by $p$, we observe 
$\chi(x)=0$. It means that $\Gamma(H)$ is connected. This is a contradiction and hence $G K(G)$ is disconnected. Then by Lemma 3.1, $O C(G)=O C(H)$. According to Table 2, $G$ is an $O C$-characterizable group with $s(G)=2$ and therefore $G \cong H$.

Lemma 3.1 will be of use to show the validity of Conjecture 1.1 for more $O C$ characterizable simple groups of Lie type that we state as a general result.

Theorem 3.1. Let $G$ and $H$ be two groups with the same order. Suppose $G$ is an $O C$-characterizable simple group of Lie type with $s(G)=2$ and $G K(H)$ is disconnected. If $\operatorname{vo}(G)=\operatorname{vo}(H)$, then $G \cong H$.

In particular, the Conjecture 1.1 is valid for any group of Table 2 with a prime $m_{2}$.

Table 1: The prime graph components of the simple groups of Lie type over the field of even characteristic.

\begin{tabular}{|l|l|l|}
\hline Type & Factors for primes in $\pi_{1}$ & $m_{2}$ \\
\hline$A_{p-1}(q),(p, q) \neq(3,2),(3,4)$ & $q, q^{i}-1,1 \leq i \leq p-1$ & $\frac{q^{p}-1}{(q-1)(q-1, p)}$ \\
$A_{p}(q), q-1 \mid p+1$ & $q, q^{p+1}-1, q^{i}-1,1 \leq i \leq p-1$ & $\frac{q^{p}-1}{q-1}$ \\
$C_{k}(q), k=2^{n}$ & $q, q^{k}-1, q^{2 i}-1,1 \leq i \leq k-1$ & $q^{k}+1$ \\
$C_{p}(q),(q-1, p)=1$ & $q, q^{p}+1, q^{2 i}-1,1 \leq i \leq p-1$ & $q^{p}-1$ \\
$D_{p}(q),(q-1, p)=1$ & $q, q^{2 i}-1,1 \leq i \leq p-1$ & $\frac{q^{p}-1}{q-1}$ \\
$D_{p+1}(2)$ & $2,2^{2 i}-1,1 \leq i \leq p-1$, & $2^{p}-1$ \\
& $2^{p}+1,2^{p+1}-1$ & \\
${ }^{2} A_{3}\left(2^{2}\right)$ & 2,3 & 5 \\
${ }^{2} A_{p-1}\left(q^{2}\right)$ & $q, q^{i}-(-1)^{i}, 1 \leq i \leq p-1$ & $\frac{q^{p}+1}{(q+1)(q+1, p)}$ \\
${ }^{2} A_{p}\left(q^{2}\right), q+1 \mid p+1$ & $q, q^{p+1}-1, q^{i}-(-1)^{i}$, & $q^{p}+1$ \\
& $1 \leq i \leq p-1$ & \\
\hline${ }^{2} D_{k}(q), k=2^{n}, n \geq 2$ & $q, q^{2 i}-1,1 \leq i \leq k-1$ & $q^{k}+1$ \\
${ }^{2} D_{k+1}(2), k=2^{n}, n \geq 2$ & $2,2^{2 i}-1,1 \leq i \leq k-1$, & $2^{k}+1$ \\
& $2^{k}-1,2^{k+1}+1$ & \\
$G_{2}(q), q \equiv 1(\bmod 3)$ & $q, q^{2}-1, q^{3}-1$ & $q^{2}-q+1$ \\
$G_{2}(q), q \equiv-1(\bmod 3)$ & $q, q^{2}-1, q^{3}+1$ & $q^{2}+q+1$ \\
${ }^{3} D_{4}\left(q^{3}\right)$ & $q, q^{6}-1$ & $q^{4}-q^{2}+1$ \\
${ }^{2} F_{4}(2)^{\prime}$ & $2,3,5$ & 13 \\
$E_{6}(q), q \equiv 1(\bmod 3)$ & $q, q^{5}-1, q^{8}-1, q^{12}-1$ & $q^{6}+q^{3}+1$ \\
$E_{6}(q), q \equiv 1(\bmod 3)$ & $q, q^{5}-1, q^{8}-1, q^{12}-1$ & $q^{6}+q^{3}+1$ \\
${ }^{2} E_{6}\left(q^{2}\right), q \equiv-1(\bmod 3)$ & $q, q^{5}+1, q^{8}-1, q^{12}-1$ & $q^{6}-q^{3}+1$ \\
${ }^{2} E_{6}\left(q^{2}\right), q \equiv 1(\bmod 3)$ & $q, q^{5}+1, q^{8}-1, q^{12}-1$ & $q^{6}-q^{3}+1$ \\
\hline
\end{tabular}


Table 2: $O C$-characterizable simple groups of Lie type with their prime graphs having two connected components.

\begin{tabular}{|c|c|c|}
\hline$G$ & Restriction on $G$ & Reference \\
\hline$A_{p-1}(q)$ & $p \neq 3, q \neq 2,4$ & {$[16,15,26]$} \\
$A_{p}(q)$ & $(q-1) \mid(p+1)$ & {$[8,34]$} \\
${ }^{2} A_{p}(q)$ & $(q+1) \mid(p+1), p \neq 3,5, q \neq 2,3$ & {$[29]$} \\
${ }^{2} A_{p-1}(q)$ & & {$[18,19,20,30]$} \\
$B_{n}(q)$ & $n=2^{m} \geq 2$, & {$[22,39,25,28]$} \\
$B_{p}(3)$ & & {$[7]$} \\
$C_{n}(q)$ & $n=2^{m} \geq 2$ & {$[22,39,25,28]$} \\
$C_{p}(q)$ & $q=2,3$ & {$[7]$ and Table 4 of $[23]$} \\
$D_{p}(5)$ & $p \geq 5, q=2,3,5$ & Table 4 of $[23]$ \\
$D_{p+1}(q)$ & $q=2,3$ & {$[6]$} \\
${ }^{2} D_{n}(q)$ & $n=2^{m}$ & {$[27,31]$} \\
${ }^{2} D_{n}(2)$ & $n=2^{m}+1, m \geq 2$ & {$[9]$} \\
${ }^{2} D_{p}(3)$ & $5 \leq p \neq 2^{m}+1$ & {$[35,5]$} \\
${ }^{2} D_{n}(3)$ & $n=2^{m}+1 \neq p, m \geq 2$ & {$[4]$} \\
${ }^{3} D_{4}(q)$ & & {$[3]$} \\
$E_{6}(q)$ & & {$[33]$} \\
${ }^{2} E_{6}(q)$ & $q>2$ & {$[32]$} \\
$F_{4}(q)$ & $q$ odd & {$[21,17]$} \\
$G_{2}(q)$ & $2<q \equiv \varepsilon(\bmod 3), \varepsilon= \pm 1$ & {$[1,2]$} \\
\hline
\end{tabular}

\section{2. $\quad$ Proof of Theorem 1.3}

From $\operatorname{vo}(G)=\operatorname{vo}(H)$ and Lemma 2.2, we deduce that $G K(G)=\Gamma(G)=\Gamma(H)$. Since for a simple group $G$ with $s(G)>2$, non-solvability of $H$ is concluded from Lemma 2.1 (b), it is sufficient that we investigate the case $s(G)=2$. Let $H$ be a solvable group and $G$ be a simple group of Lie type with $s(G)=2$. Since $\Gamma(H)$ has two connected components, Lemma 2.1 (b) implies that $H$ is either a Frobenius group or a nearly 2-Frobenius group. For both cases, using Lemma 2.1 (a), (b) and (c), $G K(G)$ has two clique connected components. So $G$ is the above mentioned simple group of Theorem 2.2. According to Tables 3 and 4 for simple groups of Lie type with $s(G)=2$ except $A_{2}(q)$, where $(q-1)_{3} \neq 3$ and $q$ is a Mersenne prime, ${ }^{2} A_{2}(q)$, where $(q+1)_{3} \neq 3$ and $q$ is a Fermat prime, $C_{2}(q)$ where $q>2,{ }^{2} A_{2}(9)$, $C_{3}(2), D_{4}(2)$ and ${ }^{3} D_{4}(2)$, we have $t(G) \geq 3$. Thus, if $p, q, r \in \rho(G)$, then at least two of them lie in a component such that they are non-adjacent, which is impossible. Now, if $G$ is one of the following groups: ${ }^{2} A_{2}(9), C_{3}(2), D_{4}(2)$ or ${ }^{3} D_{4}(2)$, then $G$ is a $K_{4}$-group and Theorem 2.1 implies $H \cong G$. Hence the desired conclusion holds. 
Table 3: Independence number and set of finite simple classical groups of Lie type.

\begin{tabular}{|c|c|c|c|}
\hline$G$ & Condition & $t(G)$ & $\rho(G)$ \\
\hline$A_{n-1}(q)$ & $\begin{array}{l}n=2, q>3 \\
n=3,(q-1)_{3}=3 \text { and } q+1 \neq 2^{k} \\
n=3,(q-1)_{3} \neq 3 \text { and } q+1 \neq 2^{k} \\
n=3,(q-1)_{3}=3 \text { and } q+1=2^{k} \\
n=3,(q-1)_{3} \neq 3 \text { and } q+1=2^{k} \\
n=4 \\
n=5,6, q=2 \\
7 \leq n \leq 11, q=2 \\
n \geq 5 \text { and } q>2 \text { or } n \geq 12 \text { and } q=2\end{array}$ & $\begin{array}{c}3 \\
4 \\
3 \\
3 \\
2 \\
3 \\
3 \\
{\left[\frac{n-1}{2}\right]} \\
\left.\frac{n+1}{2}\right] \\
\end{array}$ & $\begin{array}{c}\left\{p, r_{1}, r_{2}\right\} \\
\left\{p, 3, r_{2}, r_{3}\right\} \\
\left\{p, r_{2}, r_{3}\right\} \\
\left\{p, 3, r_{3}\right\} \\
\left\{p, r_{3}\right\} \\
\left\{p, r_{n-1}, r_{n}\right\} \\
\{5,7,31\} \\
\left\{r_{i} \mid i \neq 6,\left[\frac{n}{2}\right]<i \leq n\right\} \\
\left\{r_{i} \mid\left[\frac{n}{2}\right]<i \leq n\right\} \\
\left\{\begin{array}{l}2 \\
2\end{array} \mid<1\right.\end{array}$ \\
\hline${ }^{2} A_{n-1}(q)$ & $\begin{array}{l}n=3, q \neq 2,(q+1)_{3}=3, \text { and } q-1 \neq 2^{k} \\
n=3,(q+1)_{3} \neq 3 \text { and } q-1 \neq 2^{k} \\
n=3,(q+1)_{3}=3 \text { and } q-1=2^{k} \\
n=3,(q+1)_{3} \neq 3 \text { and } q-1=2^{k} \\
n=4, q=2 \\
n=4, q>2 \\
n=5, q=2 \\
n \geq 5 \text { and }(n, q) \neq(5,2)\end{array}$ & $\begin{array}{c}4 \\
3 \\
3 \\
2 \\
2 \\
3 \\
3 \\
{\left[\frac{n+1}{2}\right]}\end{array}$ & $\begin{array}{c}\left\{p, 3, r_{1}, r_{6}\right\} \\
\left\{p, r_{1}, r_{6}\right\} \\
\left\{p, 3, r_{6}\right\} \\
\left\{p, r_{6}\right\} \\
\{2,5\} \\
\left\{p, r_{4}, r_{6}\right\} \\
\{2,5,11\} \\
\left\{r_{i / 2} \mid\left[\frac{n}{2}\right]<i \leq n,\right. \\
i \equiv 2(\bmod 4)\} \cup \\
\left\{r_{2 i} \mid\left[\frac{n}{2}\right]<i \leq n,\right. \\
i \equiv 1(\bmod 2)\} \cup \\
\left\{r_{i} \mid\left[\frac{n}{2}\right]<i \leq n,\right. \\
i \equiv 0(\bmod 4)\}\end{array}$ \\
\hline $\begin{array}{c}B_{n}(q) \text { or } \\
C_{n}(q)\end{array}$ & $\begin{array}{l}n=2, q>2 \\
n=3, q=2 \\
n=4, q=2 \\
n=5, q=2 \\
n=6, q=2 \\
n>2,(n, q) \neq(3,2),(4,2),(5,2),(6,2)\end{array}$ & $\begin{array}{c}2 \\
2 \\
3 \\
4 \\
5 \\
{\left[\frac{3 n+5}{4}\right]}\end{array}$ & $\begin{array}{c}\left\{p, r_{4}\right\} \\
\{5,7\} \\
\{5,7,17\} \\
\{7,11,17,31\} \\
\{7,11,13,17,31\} \\
\left\{r_{2 i} \mid\left[\frac{n+1}{2}\right] \leq i \leq n\right\} \cup \\
\left\{r_{i} \mid\left[\frac{n}{2}\right]<i \leq n\right. \\
i \equiv 1(\bmod 2)\}\end{array}$ \\
\hline$\overline{D_{n}(q)}$ & $\begin{array}{l}n=4 \text { and } q=2 \\
n=5 \text { and } q=2 \\
n=6 \text { and } q=2 \\
n \geq 4 \\
(n, q) \neq(4,2),(5,2),(6,2)\end{array}$ & $\begin{array}{c}2 \\
4 \\
4 \\
{\left[\frac{3 n+1}{4}\right]}\end{array}$ & $\begin{array}{c}\{5,7\} \\
\{5,7,17,31\} \\
\{7,11,17,31\} \\
\left\{r_{2 i} \mid\left[\frac{n+1}{2}\right] \leq i<n\right\} \cup \\
\left\{r_{i} \mid\left[\frac{n}{2}\right]<i \leq n\right. \\
i \equiv 1(\bmod 2)\} \\
\left\{r_{2 i} \mid\left[\frac{n+1}{2}\right] \leq i<n\right\} \cup \\
\left\{r_{i} \mid\left[\frac{n}{2}\right] \leq i \leq n\right\} \\
\end{array}$ \\
\hline${ }^{2} D_{n}(q)$ & $\begin{array}{l}n=4 \text { and } q=2 \\
n=5 \text { and } q=2 \\
n=6 \text { and } q=2 \\
n=7 \text { and } q=2 \\
n \geq 4, n \neq 1(\bmod 4) \\
(n, q) \neq(4,2),(6,2),(7,2) \\
n>4, n \equiv 1(\bmod 4),(n, q) \neq(5,2)\end{array}$ & $\begin{array}{c}3 \\
3 \\
5 \\
5 \\
{\left[\frac{3 n+4}{4}\right]} \\
\\
{\left[\frac{3 n+4}{4}\right]}\end{array}$ & 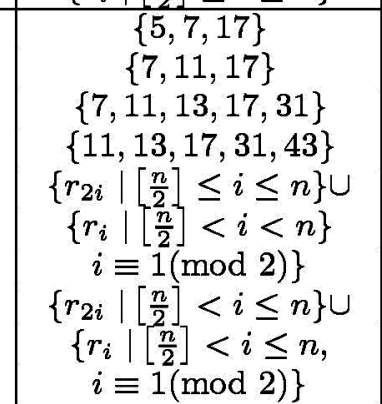 \\
\hline
\end{tabular}


Table 4: Independence number and set of finite simple exceptional Lie-type groups.

\begin{tabular}{|c|c|c|c|}
\hline$G$ & Conditions & $t(G)$ & $\rho(G)$ \\
\hline$G_{2}(q)$ & $q>2$ & 3 & $\left\{p, r_{3}, r_{6}\right\}$ \\
\hline \multirow[t]{2}{*}{$\overline{F_{4}(q)}$} & $q=2$ & 4 & $\{5,7,13,17\}$ \\
\hline & $q>2$ & 5 & $\left\{r_{3}, r_{4}, r_{6}, r_{8}, r_{12}\right\}$ \\
\hline \multirow[t]{2}{*}{$E_{6}(q)$} & $q=2$ & 5 & $\{5,13,17,19,31\}$ \\
\hline & $q>2$ & 6 & $\left\{r_{4}, r_{5}, r_{6}, r_{8}, r_{9}, r_{12}\right\}$ \\
\hline${ }^{2} E_{6}(q)$ & & 5 & $\left\{r_{4}, r_{8}, r_{10}, r_{12}, r_{18}\right\}$ \\
\hline$E_{7}(q)$ & & 7 & $\left\{r_{7}, r_{8}, r_{9}, r_{10}, r_{12}, r_{14}, r_{18}\right\}$ \\
\hline$E_{8}(q)$ & & 11 & $\left\{r_{7}, r_{8}, r_{9}, r_{10}, r_{12}, r_{14}, r_{15}, r_{18}, r_{20}, r_{24}, r_{30}\right\}$ \\
\hline \multirow[t]{2}{*}{${ }^{3} D_{4}(q)$} & $q=2$ & 2 & $\{2,13\}$ \\
\hline & $q>2$ & 3 & $\left\{r_{3}, r_{6}, r_{12}\right\}$ \\
\hline${ }^{2} B_{2}\left(2^{2 n+1}\right)$ & $n \geq 1$ & 4 & $\begin{array}{c}\left\{2, s_{1}, s_{2}, s_{3}\right\} \text { where } \\
s_{1} \mid 2^{2 n+1}-1 \\
s_{2} \mid 2^{2 n+1}-2^{n+1}+1 \\
s_{3} \mid 2^{2 n+1}+2^{n+1}+1\end{array}$ \\
\hline${ }^{2} G_{2}\left(3^{2 n+1}\right)$ & $n \geq 1$ & 5 & $\begin{array}{c}\left\{3, s_{1}, s_{2}, s_{3}, s_{4}\right\}, \text { where } \\
s_{1} \neq 2, s_{1} \mid 3^{2 n+1}-1 \\
s_{2} \neq 2, s_{2} \mid 3^{2 n+1}+1 \\
s_{3} \mid 3^{2 n+1}-3^{n+1}+1 \\
s_{4} \mid 3^{2 n+1}+3^{n+1}+1\end{array}$ \\
\hline${ }^{2} F_{4}\left(2^{2 n+1}\right)$ & $n \geq 2$ & 5 & $\begin{array}{c}\left\{s_{1}, s_{2}, s_{3}, s_{4}, s_{5}\right\}, \text { where } \\
s_{1} \neq 3, s_{1} \mid 2^{2 n+1}+1 \\
s_{2} \mid 2^{4 n+2}+1 \\
s_{3} \neq 3, s_{3} \mid 2^{4 n+2}-2^{2 n+1}+1 \\
s_{4} \mid 2^{4 n+2}-2^{3 n+2}+2^{2 n+1}-2^{n+1}+1 \\
s_{5} \mid 2^{4 n+2}+2^{3 n+2}+2^{2 n+1}+2^{n+1}+1\end{array}$ \\
\hline${ }^{2} F_{4}(2)^{\prime}$ & none & 3 & $\{3,5,13\}$ \\
\hline${ }^{2} F_{4}(8)$ & none & 4 & $\{7,19,37,109\}$ \\
\hline
\end{tabular}

\section{R E F E R E N C E S}

1. G. Y. Chen: A new characterization of $G_{2}(q)$, J. Southwest China Normal Univ., 21 (1996), 47-51.

2. G. Y. Chen: Characterization of Lie type group $G_{2}(q)$ by its order components, J. Southwest China Normal Univ., (Natural Science), 26 (5) (2001), 503-509.

3. G. Y. Chen: Characterization of ${ }^{3} D_{4}(q)$, Southeast Asian Bulletin of Math., 25, (2001), 389-401.

4. G. Y. Chen and H. SHI: ${ }^{2} D_{n}(3)\left(9 \leq n=2^{m}+1\right.$ not a prime) can be characterized by its order components, J. Appl. Math. Comput., 19, (2005), 355-362.

5. M. R. DARAFSHEH: Characterizability of group ${ }^{2} D_{p}(3)$ by its order components, where $p \geq 5$ is a prime number not of the form $2^{m}+1$, Acta Mathematica Sinica, English Series, 24 (7), (2008), 1117-1126. 
6. M. R. DARAfShen: Characterization of groups $D_{p+1}(2)$ and $D_{p+1}(3)$ using order components, J. Korean Math. Soc., 47 (2), (2010), 311-329.

7. M. R. DARAFSheH: On non-isomorphic groups with the same set of order components, J. Korean Math. Soc., 45 (1), (2008), 137-150.

8. M. R. DARAFSheh and A. MAhmini: A quantitative characterization of the linear group $L_{p+1}(2)$ where $p$ is a prime number, Kumamoto J. Math., 20, (2007), 33-50.

9. M. R. DARAfsheh and A. Mahmiani: A characterization of the group ${ }^{2} D_{n}(2)$ where $n=2^{m}+1 \geq 5$, J. Appl. Math. Comput., 31, (2009), 447-457.

10. S. Dolfi, E. PaCifici, L. SAnus and P. Spiga: On the vanishing prime graph of finite groups, J. London Math. Soc.,82 (1) (2010), 167-183.

11. S. Dolfi, E.Pacifici, L. Sanus and P. Spiga: On the vanishing prime graph of solvable groups, J. Group Theory, 13 (2010), 189-206.

12. M. Foroudi Ghasemabadi, A. Iranmanesh and F. Mavadatpour: A new characterization of some finite simple groups, Siberian Math. J., 56, (2015), 78-82.

13. M. Foroudi Ghasemabadi, A. Iranmanesh and M. Ahanjideh: A new characterization of some families of finite simple groups, Rend. Sem. Mat. Univ. Padova, European Mathematical Society, 137 (2017), 57-74.

14. N. Iiyori and H. Yamaki: Prime graph components of the simple groups of Lie type over the field of even characteristic, Proc. Japan Acad., 67, Ser. A, 1991.

15. A. Iranmanesh, S. H. Alavi and B. Khosravi: A characterization of $P S L(3, q)$ where $q$ is an odd prime power, J. Pure Appl. Algebra, 170, (2002), 243-254.

16. A. Iranmanesh, S. H. Alavi and B. Khosravi: A characterization of $\operatorname{PSL}(3, q)$ where $q=2^{n}$, Acta Math. App. Sinica (English Ser.), 18, (2002), 463-472.

17. A. Iranmanesh and B. Khosravi: A characterization of $F_{4}(q)$ where $q$ is an odd prime power, Lecture Notes London Math. Soc., 304, (2003), 277-283.

18. A. Iranmanesh and B. Khosravi: A characterization of $P S U(5, q)$, Int. Math. J., 3, (2003), 129-141.

19. A. Iranmanesh and B. Khosravi: A characterization of $P S U(7, q)$, Int. J. Appl. Math., 15, (2004), 329-340.

20. A. Iranmanesh and B. Khosravi: A characterization of $P S U(11, q)$, Canad. Math. Bull., 47, (2004), 530-539.

21. A. Iranmanesh and B. KhosRavi: A characterization of $F_{4}(q)$ where $q=2^{n},(n>1)$, Far East J. Math. Sci., (2000), 853-859.

22. A. Iranmanesh and B. KhosRavi: A characterization of $C_{2}(q)$ where $q>5$, Comment. Math. Univ. Carolin., 43, (2002), 9-21.

23. M. Khademi, M. R. DARAfsheh: Characterization of the Group $D_{p}(5)$ by order components, where $p \geq 5$ is a prime number, Southeast Asian Bulletin of Mathematics, 37 (2013), 867-885.

24. M. Khatami and A. BABAI: Recognition of some families of finite simple groups by order and set of orders of vanishing elements, Czechoslovak Math. J., 68, (2018), 121-130. 
25. B. Khosravi, B. Khosravi and B. Khosravi: The number of isomorphism classes of finite groups with the set of order components of $C_{4}(q)$, Applicable Algebra in Engineering Comunication and Computing, 15, (2005), 349-359.

26. A. Khosravi and B. Khosravi: A new characterization of $P S L(p, q)$, Comm. Algebra, 32 (6), (2004), 2325-2339.

27. A. Khosravi and B. Khosravi: A characterization of ${ }^{2} D_{n}(q)$, where $n=2^{m}$, Int. J. Math. Game Theory Algebra, 13, (2003), 253-266.

28. A. KhosRavi and B. KhosRAvi: r-Recognizability of $B_{n}(q)$ and $C_{n}(q)$ where $n=2^{m}$, J. Pure App. Algebra, (2005), 149-165.

29. A. Khosravi and B. Khosravi: A characterizability of $P S U(p+1, q)$ by its order components, Rock Mountain J. Math., 36 (5), (2006), 1555-1575.

30. B. Khosravi, B. Khosravi and B. Khosravi: A new characterization of $P S U(p, q)$, Acta Math. Hungar., 107 (3), (2005), 235-252.

31. B. Khosravi: A characterization of ${ }^{2} D_{4}(q)$, Pure Math. Appl., 12, (2001), 415-424.

32. B. Khosravi and B. Khosravi: A characterization of ${ }^{2} E_{6}(q)$, Kumamoto J. Math., 16, (2003), 1-11.

33. B. Khosravi and B. Khosravi: A characterization of $E_{6}(q)$, Algebras Groups Geom., 19, (2002), 225-243.

34. B. Khosravi and B. Khosravi: Characterizability of $\operatorname{PSL}(p+1, q)$ by its order components, Houston Journal of Mathematics, 32 (3), (2006), 683-700.

35. B. Khosravi and A. Iranmanesh: A characterization of ${ }^{2} D_{p}(3)$, where $p=2^{n}+$ $1(n \geq 2)$, Hadronic J. Suppl., 18 (4), (2003), 465-477.

36. M. Silvia Lucido and Ali Reza Moghaddamfar: Groups with complete prime graph connected components, J. Group Theory , (2004), 373-384.

37. A. V. VAsiliev and E. P. VDOvin: An adjacency criterion for the prime graph of a finite simple group, Algebra and Logic, 44 (6), (2005).

38. J. ZhANG, Z. LI and C. ShaO: Finite groups whose irreducible characters vanish only on elements of prime power order, International Electronic J. Algebra, 9 (2011), 114-123.

39. L. ZhANG and W. SHI: New characterization of $S_{4}(q)$ by its noncommuting graph, Siberian Mathematical Journal, 50 (3), (2009), 533-540.

\author{
Majedeh Pasdar \\ Faculty of Mathematical Science \\ Department of Pure Mathematics \\ P. O. Box 14115-137 \\ Tarbiat Modares University, Tehran, Iran \\ m.pasdar@modares.ac.ir
}

Ali Iranmanesh

Faculty of Mathematical Science

Department of Pure Mathematics

P.O. Box 14115-137

Tarbiat Modares University, Tehran, Iran

iranmanesh@modares.ac.ir 